\title{
Technological Trends in CFD Applications
}

\author{
Genizia Islabão de Islabão', José Carlos Costa da Silva Pinto², Ardson dos Santos Vianna Júnior³
}

\begin{abstract}
This article discusses a survey on the technology of Computational Fluid Dynamics (CFD) in various areas of knowledge and applied particularly in polymers, with scientific articles and patents as sources of information. All the data were mined using "computational", "fluid" and "dynamic" as keywords. It was observed that private companies are responsible for regulatory filings and universities, research centers and institutes hold patents related to CFD. Moreover, most of the papers selected have authors affiliated with universities and research institutes. From the found papers, $25 \%$ are related to polymers, surpassing the percentage of $10 \%$ found in the analysis of patents.
\end{abstract}

Keywords: CFD; technology monitoring.

\footnotetext{
I Instituto Militar de Engenharia, Departamento de Química, Praça General Tibúrcio, 80 - Praia. Vermelha, CEP 22290-270, Rio de Janeiro-RJ, Brasil. Email: genizia.islabao@gmail.com

2 Universidade Federal do Rio de Janeiro, Departamento de Engenharia Química, Cidade Universitária - Centro de Tecnologia, Bloco G, Sala II6, CEP 21945-970, RJ, Brasil. Email: pinto@peq.coppe.ufri.br

${ }^{3}$ Instituto Militar de Engenharia, Departamento de Química, Praça General Tibúrcio, 80 - Praia Vermelha, CEP 22290-270,

Rio de Janeiro-RJ, Brasil. Email: ardson@ime.eb.br
} 


\section{Introduction}

CFD is an acronym to Computational Fluid Dynamics, therefore, it is an issue related to computational study, mathematical or computer made, which involves fluid flows. From a CFD study it may be constructed a model that permits a better comprehension of a physical phenomenon. Generally, the CFD user is interested in velocities, pressures, and temperature fields.

As a matter of fact, CFD is one of the branches of Modeling and Simulation, which can imitate a real situation by assuming a mathematical model (Aris, 1994). For this, it can be considered basic equations, boundary conditions, and constitutive equations.

Computational Fluid Dynamics (CFD) is a computer based study - mathematical, computer made related to the dynamics of everything that flows. The CFD enables the construction of a computer based model that represents a system or device that needs to be better understood. In order to do this, it is necessary to apply the equations of fluid mechanics to evaluate a prototype, in which the answer is the fluid dynamics forecast. Basically, the CFD user is interested in having velocity distributions, pressures and temperatures in the flowing region.

The modeling and simulation process starts with the consideration of a series of assumptions related to how the physical and chemical phenomena occur within the pieces of equipment, and then, they are inserted in a series of fundamental equations (mass balance, quantity of movement and energy, conservation equations, etc.) The model is, then, mathematically solved - simulation generating answers that are compared to the data of the experiment, to its validation.

By observing the quantity of models found in the literature, the question that naturally comes up is: What makes a model "good"? Literature says that a good model is the one that optimizes the series: mathematical complexityexplanation of the results of the experiments (ARIS, 1994).

CFD involves the numerical solution of conservation equations for mass, momentum and energy in flow geometry of interest, together with additional sets of equations reflecting the problem at hand. The CFD for chemical reactor engineering is particularly important because the CFD turns out to be helpful in understanding the rule of temperature profiles and residence time distribution in determining the product quality. In this application area limitations in available computing power and experimental validation of rheological models are both important.

The comprehension of a phenomenon is made by the conceptual progress. At every new attempt, with new assumptions, there is a better understanding of what happens, more is known about the system and deeper changes can be made, as presented in Figure I.

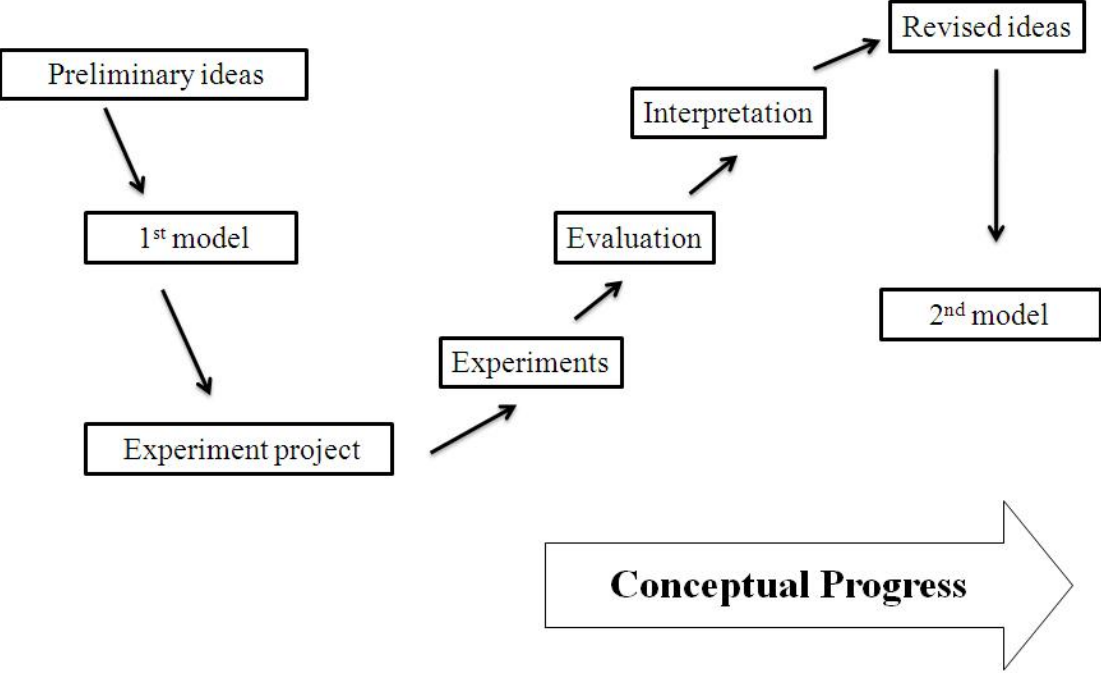

Figure I. Conceptual progress flowchart

ISSN: 07I 8-2724. (http://www.jotmi.org) 
The revision of ideas and the development of the model are not necessarily in the direction of greater complexity or in the increase in the number of parameters, but in the rescue of explanations to the phenomena and to the reproduction of experimental data (Aris, 1994). The possible profits that can be made in the use of CFD by a company may come from:

I) Insight: It's not always possible to make experiments with systems or prototypes. In these cases, the CFD analysis shows parts of the systems or phenomena, which enable assumptions to be "materialized", "tested" situations.

2) Forecast: CFD is a tool that enables to foresee what happens in case a new set of circumstances occur.

3) Efficiency: The projects and the analyses are faster and efficient, leading to shorter Project cycles. Thus, costs are reduced and you gain time.

The development of CFD can be traced back as far as the early 1900s. The pioneering efforts by Richardson (Richardson, (1910).), Courant, Friedrichs, and Lewy (Courant et. al (1928).), Southwell (Southwell, ( 1940).), von Neumann (von Neumann et. al (1950). ), Lax (Lax (1954)), as well as Godunov (Godunov (1959)) address the fundamental issues in numerical analyses for CFD (Shang, (2004)).

In their work for a typical industrial batch reactor, (Milewska et. al 2007), several "CFD computational experiments" were carried out to explore runaway and non-runaway situations. Then the results of the CFD simulations, supported by an on-line divergence criterion, have been used to detect a dangerous reactor behavior. It has been found out, that even for the reactor operated at potentially safe conditions a failure of the stirring system can lead to serious thermal runaways. The significance of a proper location of temperature probe for an early runaway detection has also been discussed. A modification of the applied divergence criterion to avoid false alarms has been proposed and tested.

Computational fluid dynamics (CFD) simulations are reported for flow, diffusion, reaction and heat transfer in a $120^{\circ}$ segment of an $N=4$ packed tube for the endothermic methane steam reforming reaction. The present work improves on previous approaches by explicit inclusion of intra-particle effects (conduction, species diffusion and reaction) coupled to realistic 3D external flow and temperature fields. It is shown that the usual assumption of symmetric species and temperature fields inside spherical catalyst particles holds for particles away from the tube wall, but particles placed in the strong temperature gradient near the tube wall show significant deviations (Dixon et. al (2007)).

In this work CFD analysis of turbulence was made in a baffled stirred tank, a three-compartment model. The results to the simulation showed that the baffle energy dissipation ratio and impeller volume ratio had no considerable change with agitation rate and impeller diameter (Vakili, (2009)).

\section{Method}

The following is the methodology adopted in this work:

(I) Before a search for documents in patent and nonpatent literature was properly set the keywords of each technology, which were incorporated in both searches, were conveniently indicated.

To the searches of patent literature documents, the corresponding IPC (International Patent Classification) codes, obtained in a pre-search, were included. Thus, the searches for patent documents employed clear criteria of indexing by the classification of the technology and the keyword, while the non-patent searches employed the same keywords only in an attempt to bring harmony and coherency to both searches.

(2) As to the databases used in the present work, all consulted were free and on-line. For example, the INPI[3], USPTO (United States Patent and Trademark Office) and EPO (European Patent Office) databases were used; related to the non-patent literature database, these were accessed through the ISI (Institute for Scientific Information) database, by means of specific web sites for scientific articles search like: SCIENCE DIRECT®, from WEB OF SCIENCE尺, and SCOPUS $尺[4]$, both accessed from a library in a public federal university.

(3) It was considered an interval of time from 1996 to 2008 , since prior to that date there was no occurrence of 
patent applications filed in the area of CFD. It is important to note that due to the phase of secrecy patent documents (18 months) the queries in these databases were in fact their main limitation of search;

(4) This way, with all the data obtained in the searches, demonstrative graphs of the results were made, mapping the evolution of the technologies related to CFD development used in the polymer area, as well as in other areas of knowledge. At the end of the implementation of this methodology, the key technologies involved, the identification of markets, and the tracking of the main countries that dominate such technological fields were assessed.

\section{Results and Discussion}

In order to draw a qualitative global picture about CFD application, patent applications filed worldwide were mapped, which allowed to identify the technological development throughout the years (Figure 2), focusing the use of this simulation tool, in the area of polymers as well as other areas of knowledge.

Figure 2 (a) presents the evolutionary behavior of the CFD technology over time in terms of patent applications filed. It was possible to observe the steady growth in the numbers of applications requested in the last years, making evident the current importance of the CFD study.

To obtain these data, a search on patent applications related to the application of CFD in the field of polymers, as well as other areas, such as process modeling and aerodynamic simulations was made. This search evaluated the period from 1996 to 2008 , for dates prior to 1996 no records of patent applications filed in the area of CFD were found.

\section{Applications $\mathbf{x}$ Time}

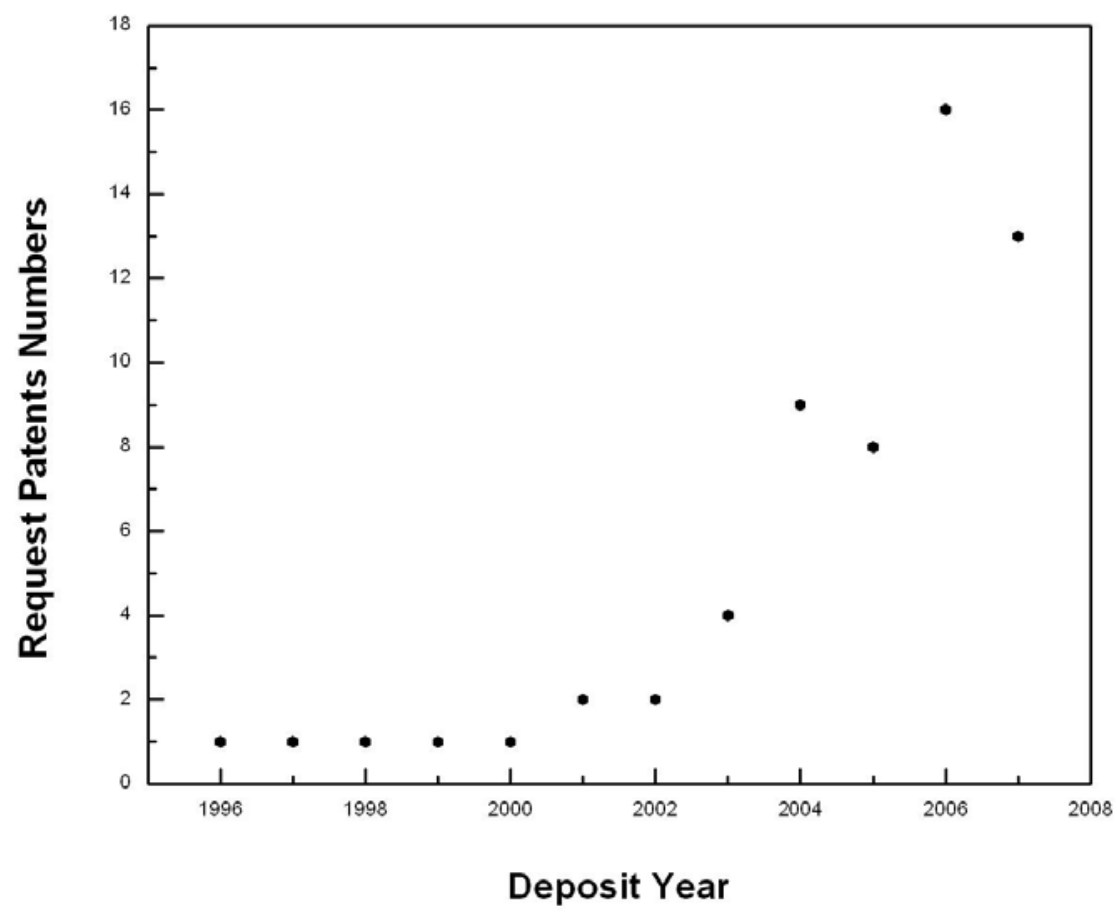

Figure 2. (a) Mapping of the Evolution of Patent Applications filing, (b) Mapping of patent applications in the countries.

Analysis of the distribution of countries which originated patented technologies show that these are mostly concentrated in more developed countries. 59 patent applications were found in the study period and the United
States appears as the main applicant of patents. From the total of patent applications, 20 were filed in the United States until 2008 as shown in Figure 2(b), which represents $34 \%$ (34 percent) of all applications filed. 


\section{Applications $x$ Countries}

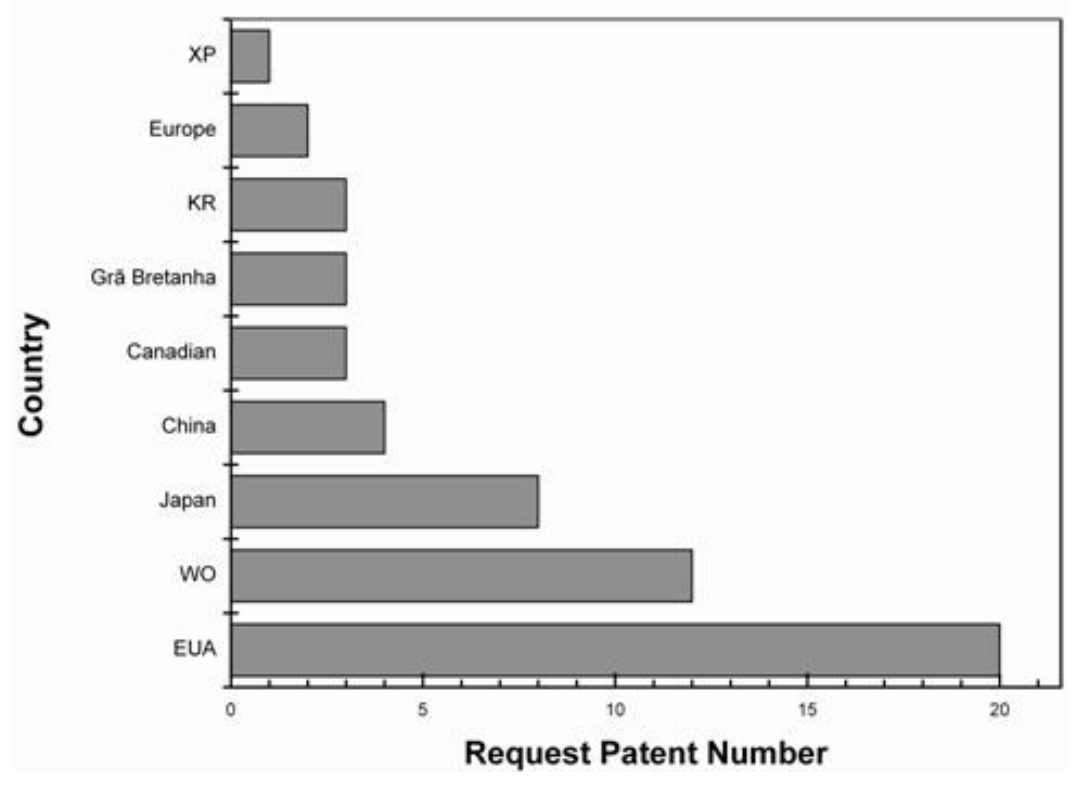

Figure 2. (b) Mapping of patent applications in the countries.

The Figure 3 shows the distribution of patent applications per year of filing, with a total of 59 applications that relate to the application of CFD, where, 10\% (10 percent) are used in the polymer and the remainder applies to different areas. The BP CHEM INT LTD, with 3 (three) patent application requests, is responsible for the filing of patents in the field of polymers.

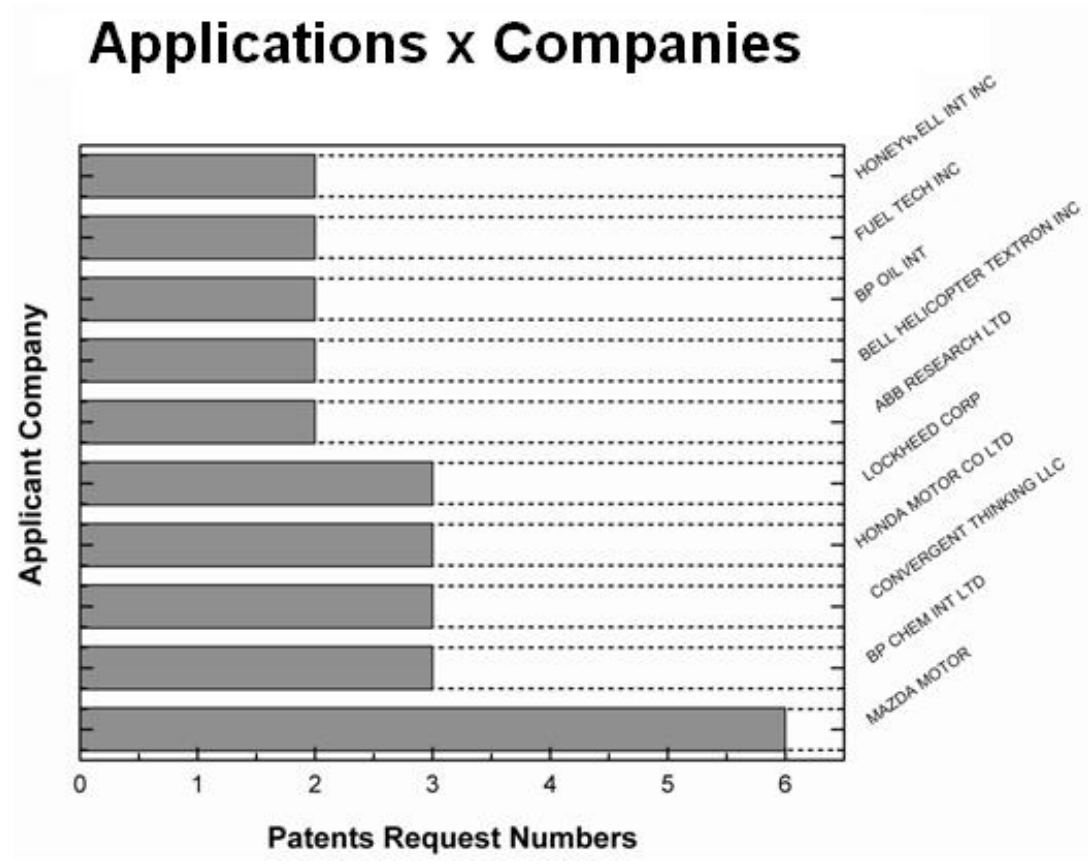

Figure 3. Mapping of Applicant Companies in CFD. 
Therefore, in view of the foregoing, it justifies the development of this work, because the area of research still has much to be explored. Modeling and simulation enables a broad comprehension of the studied phenomenon. This is the first step to the development of science and technology, without having the expenses of the experiments.

As it can be seen, private companies are responsible for regulatory filings and universities, research centers and institutes hold patents related to CFD. This seems to indicate that universities and other research centers are more focused on basic research, not including the computational fluid dynamics. Moreover, most of the papers selected have authors affiliated with universities and research institutes.

Figure 4, shows the evolutionary behavior of the CFD technology over time in terms of number of scientific articles. It was possible to observe the similar steady growth in articles with patent applications in recent years, which highlights the importance of the CFD study today.

To obtain these data, scientific articles about CFD in the field of polymers, as well as in other areas were searched. These searches included modeling processes and aerodynamic simulations. This search evaluated the period from 1991 to 2008.

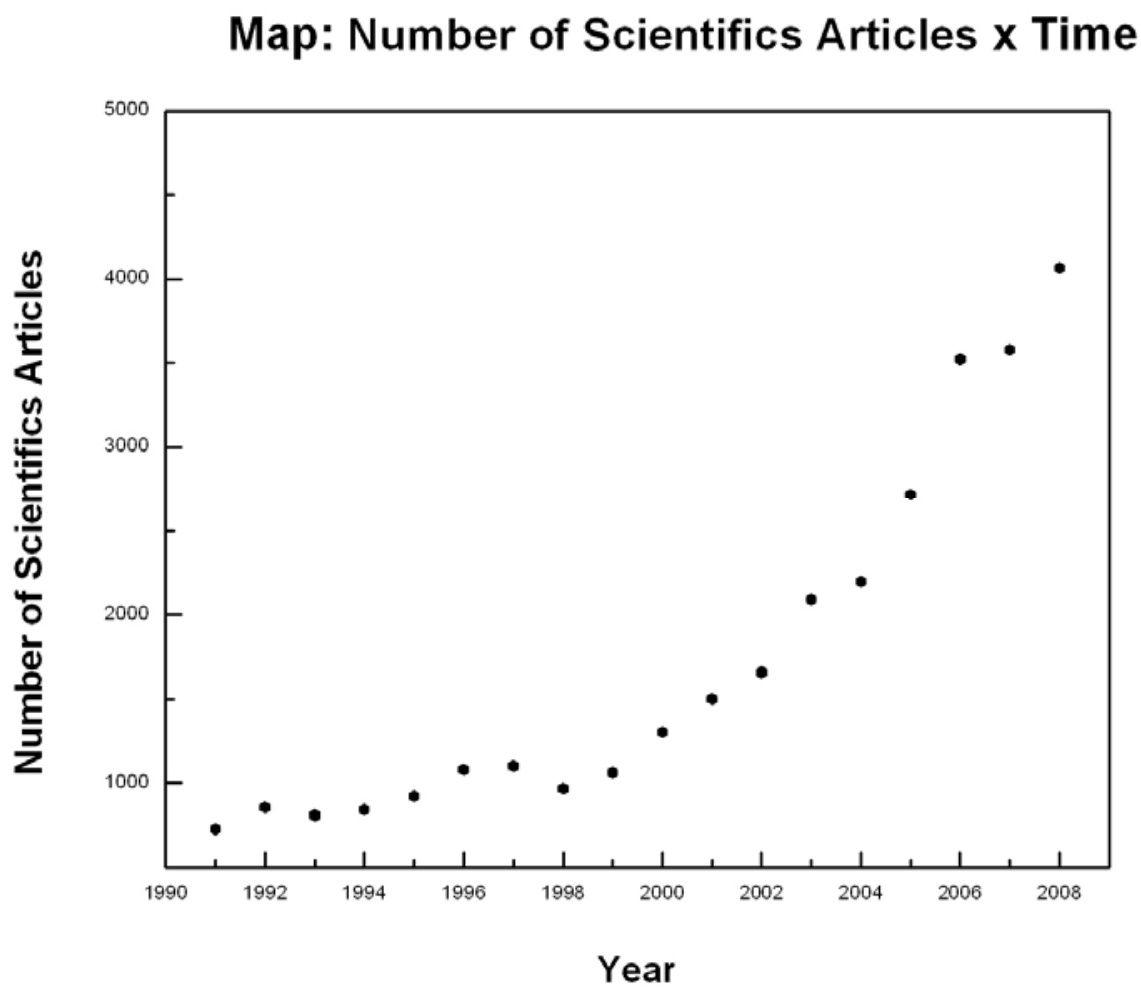

Figure 4. Mapping of the Evolution of Scientifics Articles over time.

In Figure 5, the acronyms about scientific journal mean, respectively, IJHMT (International Journal of Heat and Mass Transfer), JCP (Journal of Computational Physics), CES (Chemical Engineering Science), CMAME (Computer Methods in Applied Mechanics and Engineering) and JB (Journal of Biomechanics).
The journals most frequently used in CFD are related to the area of heat transfer and computational physics. Most scientific studies are related to the application of CFD in aerodynamics. From the papers found, $25 \%$ were related to polymers, Figure 5, surpassing the percentage of $10 \%$ found in the analysis of patents, as showed in Figure 3 above. 


\section{Number of Scientifics Articles x Newspaper}

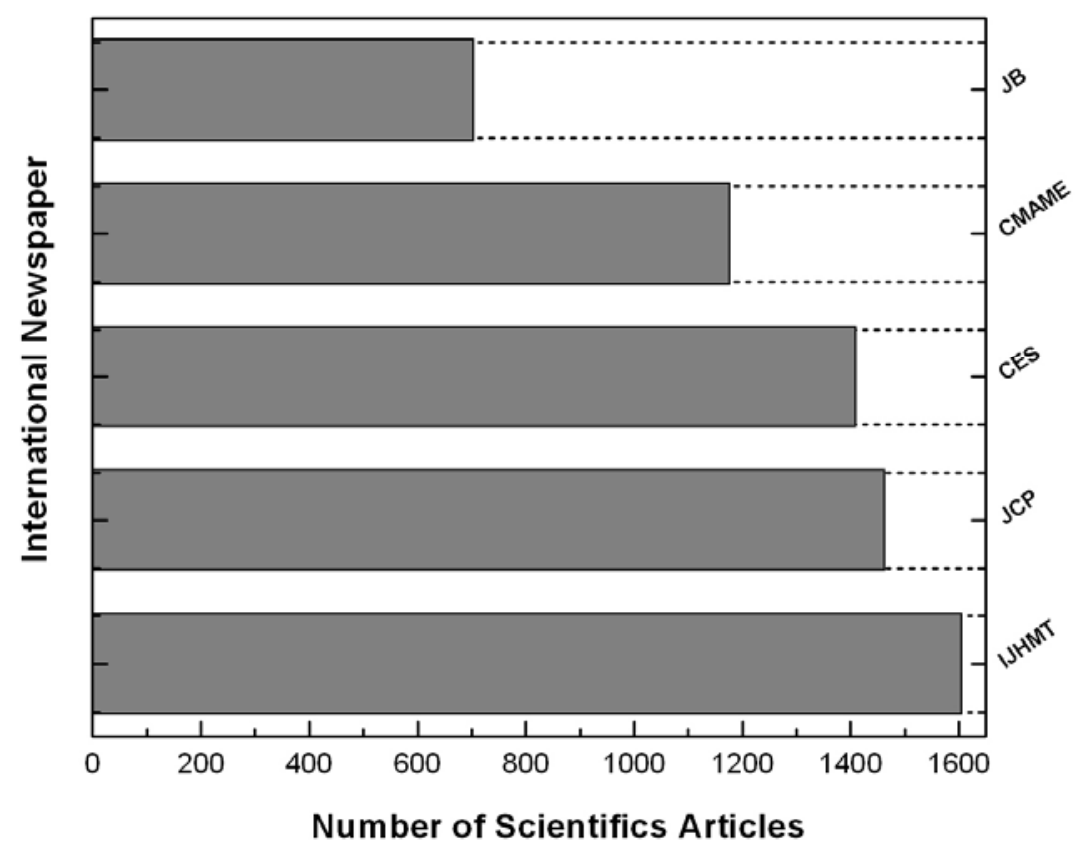

Figure 5. Mapping of the Scientific Journal and Scientifics Articles.

\section{Conclusions}

The research presented here mapped both academic documents and patent documents in the world and published in databases such as Science Direct $\AA$, Web of Science $\AA$, and SCOPUS $®$ (for articles), and sites of INPI, USPTO (United States Patent and Trademark Office) and EPO (European Patent Office) for both offices)

The study also allowed the authors to wisely indicate not only the technologies incorporated to the development of CFD, as well as companies and countries involved with the development of such systems.

The academic and patent search and investigation focused in the evolutionary character of the development of technologies related to the systems being discussed. Such approach enabled to monitor, map, and converge different pieces of information towards revealing to society a "picture" that clearly shows the emergence of new technologies, its disappearance and maintenance, as well as the obstacles and challenges surpassed in the last decade.
Private companies are responsible for applications and universities, research centers and institutes hold patents related to CFD. Moreover, most of the scientific articles selected have authors affiliated with universities and research institutes.

From the scientific articles found, $25 \%$ are related to polymers, surpassing the percentage of $10 \%$ found in the patents. From all patent applications, 20 were filed in the United States, which represents 34\% (34 percent) of total applications filed. 59 applications that relate to the application of CFD, 10\% (10 percent) are used in the polymer and the remainder applies to different areas. The BP CHEM INT LTD, with 3 (three) patent applications, is responsible for the filing of patents in the field of polymers.

In spite of allowing to foresee what happens in case a new set of circumstances occur, the application of CFD, also allows to add value to the processes, once the analyses are faster and more efficient, taking to shorter Project cycles. Thus, gaining time and reducing costs. 


\section{References}

ARIS, R. (1994). Mathematical Modelling Techniques. Dover, New York.

COURANT, R., Friedrichs, K.O., Lewy, H. (1928). Uuber die partiellen Differenzen-gleichungen der Math. Physik, Math Ann, 100, pp. 32-74.

DIXON, A.G., Taskina, M.E., Stittb, E.H., Nijemeislandb, M. (2007). 3D CFD simulations of steam reforming with resolved intraparticle reaction and gradients. Chemical Engineering Science, 62, pp. 4963- 4966.

INPI (2009). Instituto Nacional de Propriedade Industrial. http://www.inpi.gov.br. [Accessed Noveber 18, 2009]

MILEWSKA, A., Molga, E.J. (2007). CFD simulation of accidents in industrial batch stirred tank reactors. Chemical Engineering Science, 62, 4920-4925.

RICHARDSON, L.F (1910). The approximate arithmetical solution by finite differences of physical problems involving differential equations, with an application to the stresses in a masonry dam. Phil Trans $R$ Soc London, Series A; 2 10, 30757.

SCOPUS (2009). Scopus. http://www.scopus.com [Accessed October 10, 2009)]

SHANG, J.S. (2004). Three decades of accomplishments in computational fluid dynamics. Progress in Aerospace Sciences, 40, pp. $173-197$.

SOUTHWELL, R.V. (1940). Relaxation methods in engineering science. Oxford University Press, London, UK.

VAKILI, M.H., Esfahany, M.N. (2009). CFD analysis of turbulence in a baffled stirred tank, a threecompartmentmodel. Chemical Engineering Science, 64, Pp. 35I-362.

VON NEUMANN, J, Richtmeyer, R.D. (1950). A method for the numerical calculation on the hydrodynamic shocks. J Appl Phys, 2I, pp. 232-7.

LAX, P.D. (1954). Weak solution of nonlinear hyperbolic equations and their numerical computation. Commun Pure Appl Math, 7, pp. 159-63.
GODUNOV, S.K. (1959). Finite-difference method for numerical computational of discontinuous solution of the equations of fluid dynamics. Mat Sb, 47, pp. 27I-306. 